\title{
THREE-PARAMETER AND FOUR-PARAMETER LINEAR FAMILIES OF CONICS IN THE GALOIS FIELDS OF ORDER $2^{n *}$
}

\author{
BY A. D. CAMPBELL
}

1. Three-Parameter Families. We shall denote a Galois field of order $2^{n}$ by the symbol $G F\left(2^{n}\right)$. We define a threeparameter linear family of conics in such a $G F\left(2^{n}\right)$ as the locus of all points whose coordinates $x, y, z$ satisfy an equation of the form

$$
\lambda C_{1}+\mu C_{2}+\nu C_{3}+\rho C_{4}=0
$$

where

$$
\begin{aligned}
& C_{i}=a_{i} x^{2}+b_{i} y^{2}+c_{i} z^{2}+f_{i} y z+g_{i} z x+h_{i} x y=0, \\
&(i=1,2,3,4),
\end{aligned}
$$

and where the variables, coefficients, and parameters represent numbers in this domain. The conics $C_{1}=0, \cdots, C_{4}=0$ are linearly independent, and are called fundamental conics of this family. In this paper we derive the classes of these families, and we give a typical family for each class. We note that in any $G F\left(2^{n}\right)$ every number is a perfect square with just one square root, and $(\alpha x+\beta y+\gamma z)^{2}=\alpha^{2} x^{2}+\beta^{2} y^{2}+\gamma^{2} z^{2}$. We note that every such family has at least one double line.

We first divide these families into the following distinct sets.

Set I. Each family contains a net of conics reducible to the form

$$
\lambda x^{2}+\mu y^{2}+\nu z^{2}=0 .
$$

Set II. Each family contains no net reducible to (2), but does contain a net reducible to the form

$$
\lambda x^{2}+\mu y^{2}+2 \nu x y=0 .
$$

\footnotetext{
* Presented to the Society, December 29, 1923.
} 
Set III. Each family contains at least two double lines, but no net reducible to (2) or (3).

Set IV. Each family contains only one double line, but has also a net reducible to the form

$$
\lambda x^{2}+\mu x y+\nu x z=0 .
$$

Set V. Each family has none of the preceding properties, but has a pencil reducible to the form

$$
\lambda x^{2}+\mu x y=0 \text {. }
$$

Set VI. Each family has just one double line, but none of the preceding degenerate pencils and nets.

Set I. Such a family can be put in the form

$$
\lambda x^{2}+\mu y^{2}+\nu z^{2}+\rho(f y z+g z x+h x y)=0 .
$$

We easily get

(6) Class (1): $\quad \lambda x^{2}+\mu y^{2}+\nu z^{2}+\rho x y=0$.

Set II. We obtain

(7) Class (2): $\lambda x^{2}+\mu y^{2}+\nu x y+\rho\left(z^{2}+z x\right)=0$.

(8) Class (3): $\quad \lambda x^{2}+\mu y^{2}+\nu x y+\rho z x=0$.

Any transformation

$$
\begin{gathered}
x=\alpha_{1} x^{\prime}+\beta_{1} y^{\prime}+\gamma_{1} z^{\prime}, \quad y=\alpha_{2} x^{\prime}+\beta_{2} y^{\prime}+\gamma_{2} z^{\prime}, \\
z=\alpha_{3} x^{\prime}+\beta_{3} y^{\prime}+\gamma_{3} z^{\prime},
\end{gathered}
$$

where $\left|\alpha_{1}, \beta_{2}, \gamma_{3}\right| \neq 0$, that is to send (7) into (8) must have $\gamma_{1}=\gamma_{2}=0, \gamma_{3} \neq 0$, since the net $\rho=0$ of one family must go into that of the other. But (9) then cannot send (7) into a family lacking the term in $z^{2}$.

Set III. We can reduce such a family to the form

$$
\lambda x^{2}+\mu y^{2}+\nu x z+\rho\left(c z^{2}+f y z+h x y\right)=0 .
$$

We get the following three classes, according as $c f \neq 0$; or $c=0$, $f \neq 0$; or $c \neq 0, f=0$. 
(10) Class (4): $\lambda x^{2}+\mu y^{2}+\nu z x+\rho\left(z^{2}+y z\right)=0$.

(11) Class (5): $\quad \lambda x^{2}+\mu y^{2}+\nu z x+\rho y z=0$.

An argument similar to that used in the study of (7) and (8) shows that (10) and (11) are non-equivalent. Here the pencil $\nu=\rho=0$ of (10) must go into the similar pencil of (11) by any p ojectivity (9).

(12) Class (6): $\quad \lambda x^{2}+\mu y^{2}+\nu z x+\rho\left(z^{2}+x y\right)=0$.

t is cas to pove (12) non-ecuivalent to (10) oi (11).

$\therefore t$ Vic put (1) in t c fo: $n$.

$$
\lambda x^{2}+\mu x y+\nu x z+\rho\left(b y^{2}+c z^{2}+f y z\right)=0 .
$$

Ve get tu o classes, acco ding as $C_{4}=0$ in tl e atove fan ily is a pai. of $\_\in a l$ or conjugate imaginaı y lines.

$$
\text { Class (7): } \quad \lambda x^{2}+\mu x y+\nu x z+\rho y z=0 .
$$

$$
\text { Class (8): } \lambda x^{2}+\mu x y+\nu x z+\rho\left(y^{2}+z^{2}+\alpha y z\right)=0,
$$

whe e $C_{4}=0$ is in educible. Any transformation (9) that is to scnd (14) into (13) must tave $\beta_{1}=\gamma_{1}=0$. But then the conic $C_{4}=0$ of (14) goes into a conic $y^{\prime 2}\left(\beta_{2}{ }^{2}+\beta_{3}{ }^{2}+\alpha \beta_{2} \beta_{3}\right)+z^{\prime 2}\left(\gamma_{2}{ }^{2}\right.$ $\left.+\gamma_{3}{ }^{2}+\alpha \gamma_{2} \gamma_{3}\right)+\cdots=0$. So we must have $\beta_{2}{ }^{2}+\beta_{3}{ }^{2}+\alpha \beta_{2} \beta_{3}=0$, $\gamma_{2}^{2}+\gamma_{3}^{2}+a \gamma_{2} \gamma_{3}=0$ wlich give us $\beta_{2}=\beta_{3}=\gamma_{2}=\gamma_{3}=0$ and (9) is singular.

Set V. We can put the family in one of the three following forms:

$$
\begin{aligned}
& \lambda x^{2}+\mu x y+\nu\left(z^{2}+z x\right)+\rho\left(b y^{2}+f y z+g z x\right)=0 \\
& \lambda x^{2}+\mu x y+\nu y z+\rho\left(b y^{2}+c z^{2}+g z x\right)=0 \\
& \lambda x^{2}+\mu x y+\nu\left(z^{2}+y z\right)+\rho\left(b y^{2}+c z^{2}+g z x\right)=0 .
\end{aligned}
$$

Case (A) gives us two classes.

$$
\begin{aligned}
& \text { Class (9): } \lambda x^{2}+\mu x y+\nu\left(z^{2}+z x\right)+\rho\left(y^{2}+y z\right)=0 . \\
& \text { Class (10): } \lambda x^{2}+\mu x y+\nu\left(z^{2}+z x\right)+\rho y z=0 .
\end{aligned}
$$

Any projectivity (9) that is to send (15) into (16) must have $\beta_{1}=\gamma_{1}=\gamma_{2}=0, \beta_{2} \gamma_{3} \neq 0$. But then $C_{3}=0$ and $C_{4}=0$ of (10) 
must go separately into conics lacking the term in $y^{2}$. This gives us $\beta_{3}{ }^{2}=0, \beta_{2}{ }^{2}+\beta_{2} \beta_{3}=0$; hence $\beta_{2}=0$.

Case (B) gives us two classes.

$$
\begin{aligned}
& \text { Class (11): } \lambda x^{2}+\mu x y+\nu y z+\rho\left(y^{2}+z^{2}+z x\right)=0 . \\
& \text { Class (12): } \lambda x^{2}+\mu x y+\nu y z+\rho\left(y^{2}+z x\right)=0 .
\end{aligned}
$$

The families (17) and (18) are easily proved to be nonequivalent. They are non-equivalent to families (15) and (16) because of the presence in these two latter families of the degenerate pencil $\lambda x^{2}+\nu\left(z^{2}+z x\right)=0$.

Case (C) gives us the following class.

$$
\text { Class (13): } \quad \lambda x^{2}+\mu x y+\nu\left(z^{2}+y z\right)+\rho\left(y^{2}+\alpha z^{2}+z x\right)=0 .
$$

Set VI. We can put the family in one of two forms

$$
\begin{array}{r}
\lambda x^{2}+\mu\left(y^{2}+x y\right)+\nu y z+\rho\left(b y^{2}+c z^{2}+g z x\right)=0, \\
\lambda x^{2}+\mu y z+\nu\left(b y^{2}+c z^{2}+g z x\right) \\
+\rho\left(b^{\prime} y^{2}+c^{\prime} z^{2}+h^{\prime} x y\right)=0 .
\end{array}
$$

Case (A) gives us

$$
\text { Class (14) : }
$$

$\lambda x^{2}+\mu\left(y^{2}+x y\right)+\nu y z+\rho\left(y^{2}+\alpha z^{2}+z x\right)=0, \quad \alpha \neq 0$.

Case (B) gives us

$$
\text { Class (15) : }
$$

$$
\lambda x^{2}+\mu y z+\nu\left(y^{2}+\alpha z^{2}+z x\right)+\rho\left(\beta y^{2}+\gamma z^{2}+x y\right)=0,
$$

where $\gamma=1$ or $\gamma \neq$ cube, $\gamma / \beta \neq \alpha$. The family (20) has a degenerate pencil $\lambda x^{2}+\mu\left(y^{2}+x y\right)=0$, which does not occur in (21).

2. Four-Parameter Families. We define a four-parameter linear family of conics in a $G F\left(2^{n}\right)$ by the equation

$$
\lambda C_{1}+\mu C_{2}+\nu C_{3}+\rho C_{4}+\sigma C_{5}=0,
$$

where the details of notation are as indicated in the description of equation (1). It is easy to show that every such family has at least two double lines. First we assume that 
(22) has a degenerate net reducible to (2). Then we assume that (22) has no net (2), but a net (3). Then we assume that (22) has neither (2) nor (3).

If (22) has a net (2) we can put the family in the form

$$
\lambda x^{2}+\mu y^{2}+\nu z^{2}+\rho x y+\sigma(f y z+g z x)=0,
$$

which gives us

$$
\text { Class (1): } \quad \lambda x^{2}+\mu y^{2}+\nu z^{2}+\rho x y+\sigma x z=0 .
$$

If (22) has a net (3), but not a net (2), we get

$$
\lambda x^{2}+\mu y^{2}+\nu x y+\rho x z+\sigma\left(c z^{2}+f y z\right)=0,
$$

which gives us, according as $c \neq 0$, or $c=0$,

$$
\text { Class (2): } \quad \lambda x^{2}+\mu y^{2}+\nu x y+\rho x z+\sigma\left(z^{2}+y z\right)=0,
$$

$$
\text { Class (3): } \quad \lambda x^{2}+\mu y^{2}+\nu x y+\rho x z+\sigma y z=0 .
$$

If (9) is to send (24) into (25) we must have $\gamma_{1}=\gamma_{2}=0$, $\gamma_{3} \neq 0$. But such a transformation cannot send (24) into a family lacking the term in $z^{2}$.

If (22) has no net (2) and no net (3) we easily reduce the family to the form

(26) Class (4): $\lambda x^{2}+\mu y^{2}+\nu x z+\rho y z+\sigma\left(z^{2}+x y\right)=0$.

Syracuse University 